\title{
The Parkinson's disease protein LRRK2 impairs proteasome substrate clearance without affecting proteasome catalytic activity
}

\author{
M Lichtenberg ${ }^{1,2}$, A Mansilla ${ }^{1,2}$, VR Zecchini ${ }^{1,2}$, A Fleming $^{1,2}$ and DC Rubinsztein ${ }^{*, 1}$
}

Leucine-rich repeat kinase 2 (LRRK2) mutations are the most common known cause of Parkinson's disease (PD). The clinical features of LRRK2 PD are indistinguishable from idiopathic PD, with accumulation of $\alpha$-synuclein and/or tau and/or ubiquitin in intraneuronal aggregates. This suggests that LRRK2 is a key to understanding the aetiology of the disorder. Although loss-offunction does not appear to be the mechanism causing PD in LRRK2 patients, it is not clear how this protein mediates toxicity. In this study, we report that LRRK2 overexpression in cells and in vivo impairs the activity of the ubiquitin-proteasome pathway, and that this accounts for the accumulation of diverse substrates with LRRK2 overexpression. We show that this is not mediated by large LRRK2 aggregates or sequestration of ubiquitin to the aggregates. Importantly, such abnormalities are not seen with overexpression of the related protein LRRK1. Our data suggest that LRRK2 inhibits the clearance of proteasome substrates upstream of proteasome catalytic activity, favouring the accumulation of proteins and aggregate formation. Thus, we provide a molecular link between LRRK2, the most common known cause of PD, and its previously described phenotype of protein accumulation.

Cell Death and Disease (2011) 2, e196; doi:10.1038/cddis.2011.81; published online 25 August 2011

Subject Category: Experimental Medicine

Parkinson's disease (PD) is the second most common human neurodegenerative disease. PD pathology involves loss of dopaminergic neurons in the substantia nigra pars compacta and other regions, and the formation of Lewy bodies (LB) containing $\alpha$-synuclein. ${ }^{1}$ Excessive $\alpha$-synuclein is toxic, as rare cases of inherited PD are caused by $\alpha$-synuclein gene triplication. ${ }^{2}$ However, the mechanisms causing $\alpha$-synuclein accumulation in sporadic PD are unclear.

Mutations in leucine-rich repeat kinase 2 (LRRK2), the most common known cause of autosomal dominant PD, are also found in 'sporadic' cases. The neurological and neurochemical phenotypes are indistinguishable from the idiopathic form of PD. ${ }^{3-5}$ Brains from PD patients with $L R R K 2$ mutations have accumulation of $\alpha$-synuclein and/or tau in intraneuronal aggregates as well as ubiquitin-positive inclusions. ${ }^{3}$ This variable pathology suggests that LRRK2 acts far upstream in the PD neurodegeneration cascade.

The protein inclusions in PD patients may be due to failure of the two major intracellular protein breakdown pathways, the ubiquitin-proteasome system (UPS) and macroautophagy. In the UPS, E1, E2 and E3 enzymes tag proteins with four or more ubiquitin molecules, which target the substrate to the proteasome, a barrel-shaped multiprotein complex with three different protease activities that degrade substrates into peptides. In macroautophagy, a double-layered membrane forms around a portion of cytosol. This autophagosome fuses with lysosomes, where its contents are degraded.

The LRRK2 protein has 2527 amino acids and several independent domains, including three protein-protein-interacting domains (ankyrin, LRR and WD40) and two enzymatic activities (kinase and GTPase). ${ }^{6}$ The various mutations associated with PD are found throughout the protein. Knockout studies in mice suggest that LRRK2 mutations confer a gain-of-function. ${ }^{7,8}$ Initially, increased kinase activity was considered as the pathogenic gain-of-function mechanism. $^{9,10}$ However, subsequent studies confirmed this finding only for the commonest LRRK2 mutation, G2019S. Results for other mutations have been contradictory and other toxic effects may therefore exist. ${ }^{11}$

In this study, we investigated the role of LRRK2 in protein homoeostasis. We observed $\alpha$-synuclein and ubiquitin accumulation in LRRK2-expressing cells and in a new LRRK2 zebrafish model. This imbalance in protein homoeostasis is not only restricted to $\alpha$-synuclein but can also affect a range of

\footnotetext{
'Department of Medical Genetics, University of Cambridge, Cambridge Institute for Medical Research, Addenbrooke's Hospital, Cambridge CB2 0XY, UK ${ }^{*}$ Corresponding author: DC Rubinsztein, Department of Medical Genetics, University of Cambridge, Cambridge Institute for Medical Research, Addenbrooke's Hospital, Hills Road, Cambridge CB2 OXY, UK. Tel: + 44(0)1223 762 608; Fax: + 44(0)1223 331 206; E-mail: dcr1000@ hermes.cam.ac.uk

${ }^{2}$ These authors are joint first authors.

Keywords: Parkinson's disease; ubiquitin-proteasome system; LRRK2; aggregation

Abbreviations: LRRK2, leucine-rich repeat kinase 2; PD, Parkinson's disease; LB, lewy body; UPS, ubiquitin-proteasome system; CMA, chaperone-mediated autophagy; DUB, de-ubiquitinating enzymes; WTLRRK2, wild-type LRRK2; GSLRRK2, G2019S mutated LRRK2; httQ23, huntingtin wild-type exon 1; httQ74, huntingtin exon 1 with polyglutamine expansion; KMLRRK2, kinase-dead K1906M mutation in LRRK2; MEF, mouse embryonic fibroblasts; HSP70, heat-shock protein 70; NAC, $N$-acetyl-L-cysteine; CHX, cycloheximide; h.p.f., hours post-fertilisation

Received 05.7.11; accepted 20.7.11; Edited by M Piacentini
} 
a - vector - GSLRRK2

- WTLRRK2 - httQ23
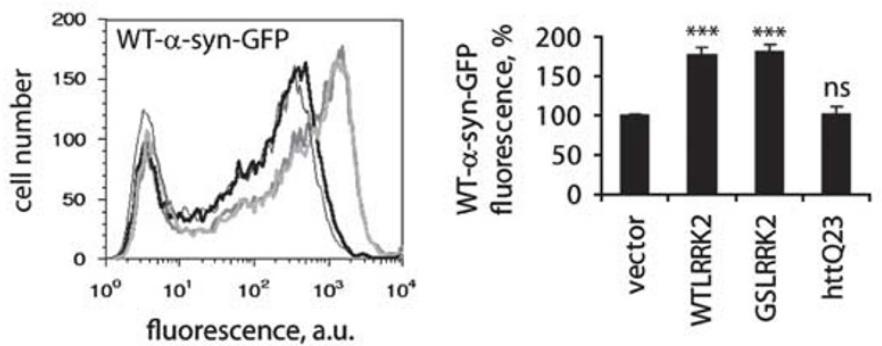

b
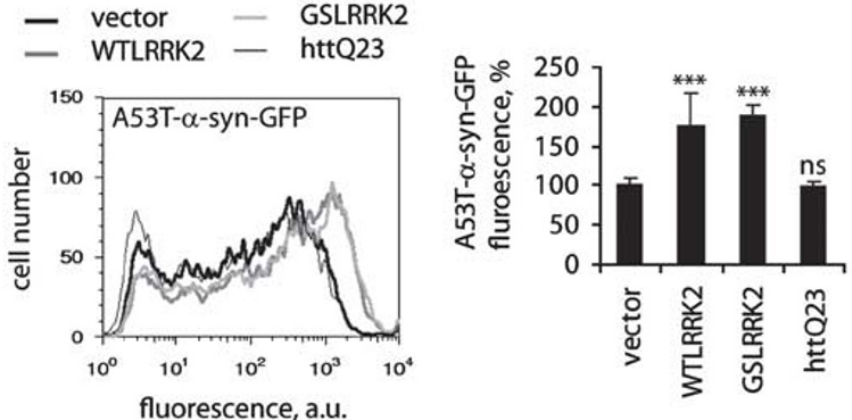

e
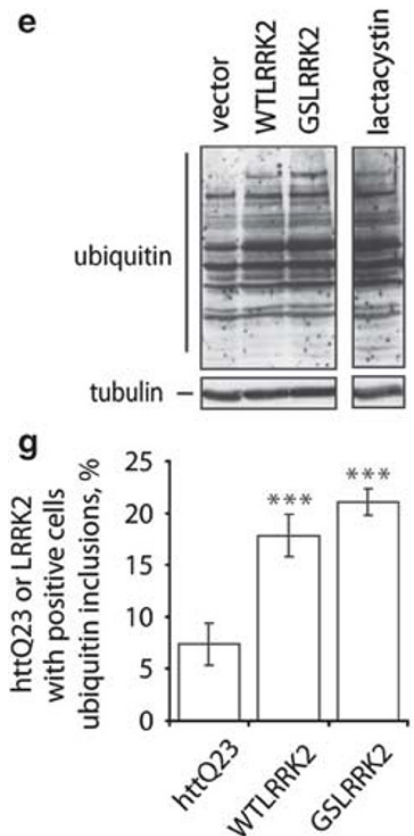

c

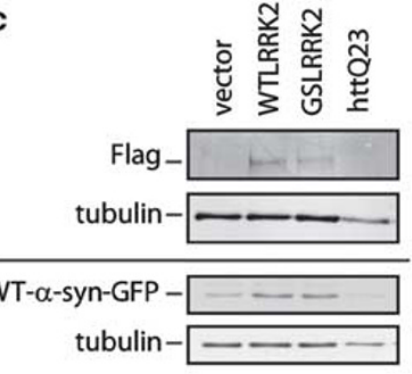

d

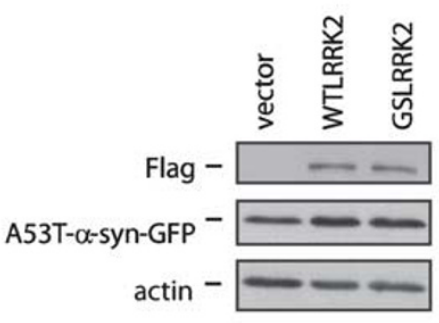

WTLRRK2

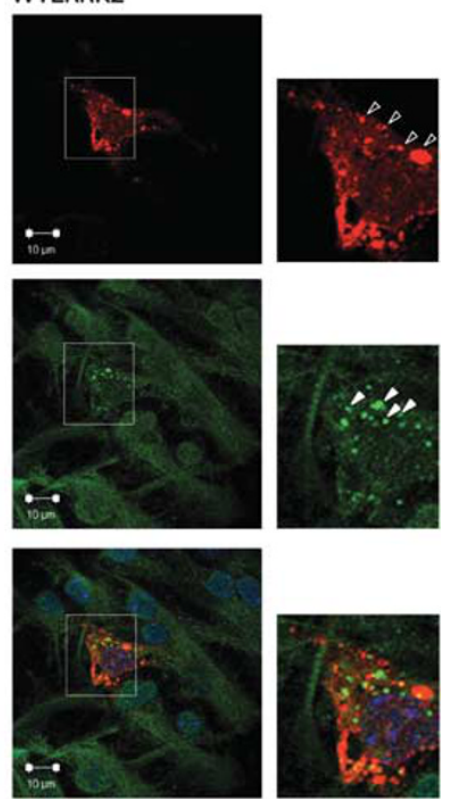

Figure 1 LRRK2 overexpression leads to increased protein levels. (a) GFP-tagged wild-type $\alpha$-synuclein or (b) GFP-tagged A53T- $\alpha$-synuclein was co-transfected in HeLa cells with either empty vector, httQ23, WTLRRK2 or GSLRRK2. Increased GFP fluorescence was detected in cells overexpressing LRRK2 $48 \mathrm{~h}$ post-transfection by flow cytometry. Left: representative flow cytometry histograms show fluorescence intensity plotted against cell number. Increased fluorescence is observed in WTLRRK2- and GSLRRK2-transfected cells (dark grey and light grey line, respectively), compared with cells transfected with vector (black line) or httQ23 (wild-type huntingtin exon 1, used as a control) (thin black line); right: quantification of (a) and (b). Graphs show geometric mean of cellular fluorescence (normalised to vector-transfected cells within each experiment) \pm S.D. of all replicates. Unpaired, two-tailed Student's $t$-test was performed on raw replicate data from individual experiments and representative $P$-values are shown. ${ }^{* \star} P<0.005 ; n s=$ not significant, $N=3$. (c and d) Cell lysates from ( $\mathbf{a}$ and $\mathbf{b}$ ) were analysed by western blotting. Increased levels of GFP-tagged WT- $\alpha$-synuclein and A53T- $\alpha$-synuclein protein were detected in LRRK2-overexpressing cells. (e) Immunoblot of LRRK2-overexpressing HeLa cell lysates showing an increase in the total amounts of ubiquitinated proteins compared with cells transfected with empty vector. Lactacystin, a proteasome inhibitor, caused an even greater increase in ubiquitinated proteins than LRRK2. The lane for lactacystin was run on the same blot but next to other lysates, which were cut from this picture. (f) Cortical neurons from E18 mouse brains were transfected with HA-httQ23 or Flag-WTLRRK2 and immunostained with antiubiquitin (green) and antiHA (red) or antiFlag (red) antibodies, respectively. Representative images were taken with a Zeiss LSM510 confocal microscope. LRRK2 aggregates (open arrow heads) and ubiquitin inclusions (filled arrow heads) do not always co-localise. Scale bars, $10 \mu \mathrm{M}$. (g) Ubiquitin inclusions are more frequently observed in neurons expressing WTLRRK2 or GSLRRK2, compared with cells expressing httQ23. Ubiquitin inclusion formation was assayed in three independent experiments. Representative data from one experiment are shown as percentage of transfected cells with ubiquitin inclusions \pm S.D. and were analysed by unconditional logistical regression analysis. ${ }^{* \star *} P<0.001, N=3$. See also Supplementary Figure S1 
a
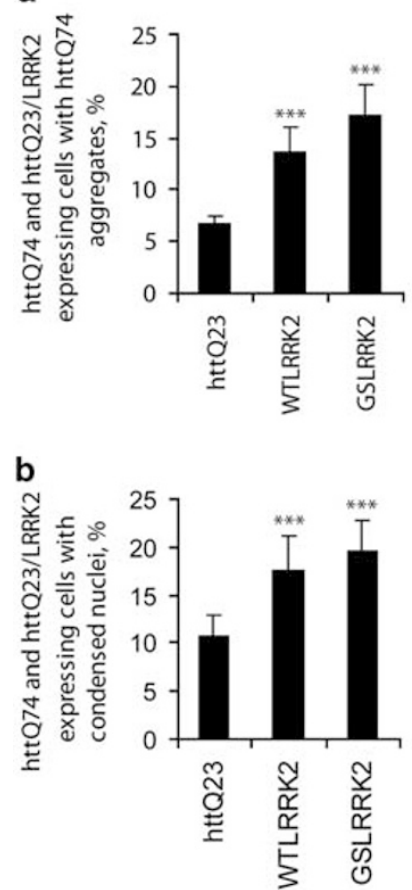

f

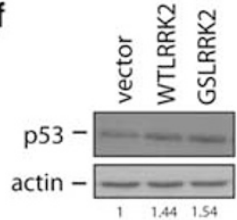

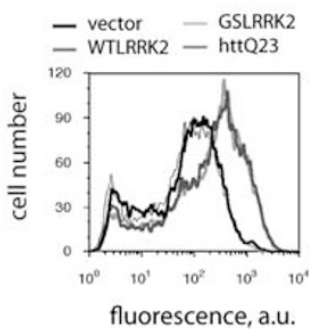

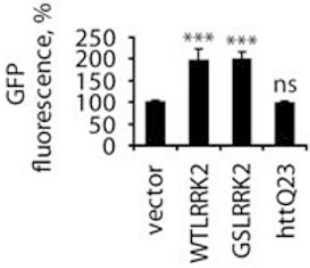

e

d

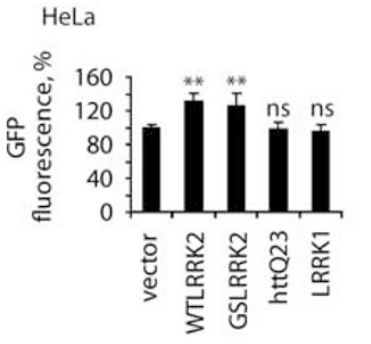

SHSY5Y

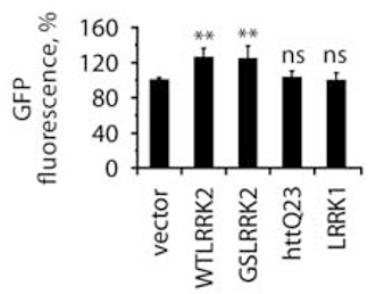

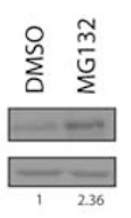

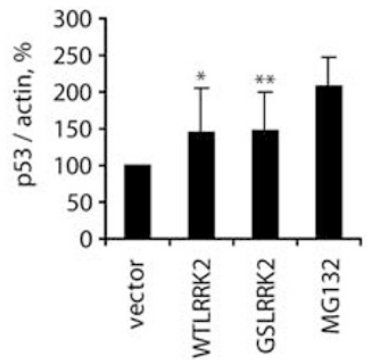

Figure 2 LRRK2 promotes httQ74 toxicity and accumulation of endogenous proteins. (a and $\mathbf{b})$ COS-7 cells were co-transfected with the aggregate-prone protein httQ74EGFP along with LRRK2 (Flag) or httQ23. Cells were fixed $48 \mathrm{~h}$ after transfection and stained with an antibody against Flag and the nuclear dye DAPI. (a) Aggregation of httQ74-EGFP and (b) cell death were scored in 200 cells per replicate. Graphs show means \pm S.E.M. of three independent experiments performed in triplicates. Statistical analysis was performed by unconditional logistical regression analysis. ${ }^{* \star \star} P<0.001, N=3$. (c) HeLa cells were transiently co-transfected with GFP along with either empty vector, httQ23, WTLRRK2 or GSLRRK2. After $48 \mathrm{~h}$ of transfection, cellular GFP fluorescence was measured by flow cytometry. Left: representative flow cytometry histogram shows that GFP fluorescence was increased in HeLa cells that expressed WTLRRK2 or GSLRRK2 (dark grey and light grey line, respectively), compared with cells transfected with vector or httQ23 (black and thin black line, respectively). Right: quantification of cellular fluorescence of three independent experiments performed in triplicates. The geometric mean of cellular fluorescence was normalised to vector-transfected cells within each experiment, error bars represent standard deviation of all replicates. Unpaired, two-tailed Student's $t$-test was performed on raw replicate data from each individual experiment and representative $P$-values are shown. ns $=$ not significant, ${ }^{* \star \star} P<0.005$, $N=3$. (d) HeLa cells were transiently co-transfected as in (c). Additionally, the effect of LRRK1 overexpression was tested on levels of GFP. Overexpression of LRRK2 but not LRRK1 or httQ23 lead to increased fluorescence, compared with vector-transfected cells. Graph represents data as above from three independent experiments performed in triplicates $(N=3)$. (e) The human neuroblastoma cell line SHSY5Y was transfected and analysed as HeLa cells in (d). Increased GFP fluorescence was measured in cells transfected with LRRK2, but not in httQ23- or LRRK1-transfected cells. Graph represents data as above from four independent experiments performed in triplicates ( $N=4$ ). (f) HeLa cells were transfected with GFP and DsRed and either control DNA or LRRK2. After $48 \mathrm{~h}$ of transfection cells were sorted for GFP and DsRed double-positives and grown for $24 \mathrm{~h}$ before cell collection. An additional vector control was treated for $12 \mathrm{~h}$ with MG132, a proteasome inhibitor, before collection. p53 levels were analysed by western blotting. Left: blots represent one of seven independent experiments. Densitometry results beneath the blots are normalised to actin. Right: quantification of seven independent experiments. Data are normalised to vector-transfected cells and graphs show mean \pm S.D. Raw data were analysed by two-tailed, paired Student's $t$-test. ${ }^{\star} P<0.05,{ }^{*} P<0.01, N=7$. Note, that MG132 treatment was only performed in two of the seven experiments

exogenous as well as endogenous proteins. Increased protein levels were independent of protein production, sequestration or impairment of autophagy but can be explained by UPS compromise. The effect of LRRK2 on the UPS is independent of its kinase activity, oxidative stress or p62. However, preventing the aggregation of LRRK2 by HSP70 can modulate the effect of LRRK2 on the UPS.

\section{Results}

Accumulation of $\alpha$-synuclein and ubiquitin in LRRK2 overexpressing cells. PD is characterised by the accumulation and aggregation of $\alpha$-synuclein. We therefore initially tested the effect of LRRK2 on $\alpha$-synuclein levels. As LRRK2 mutations are considered to cause toxicity through a 


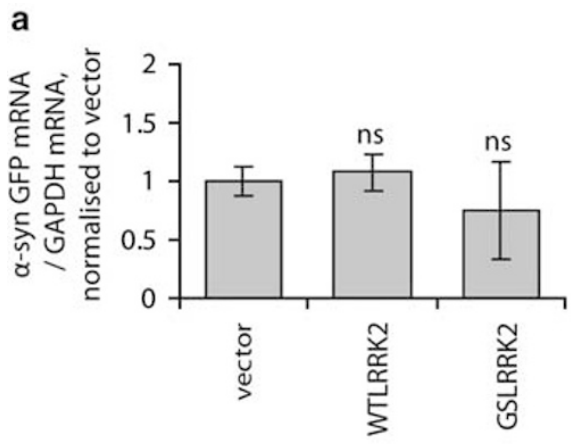

b

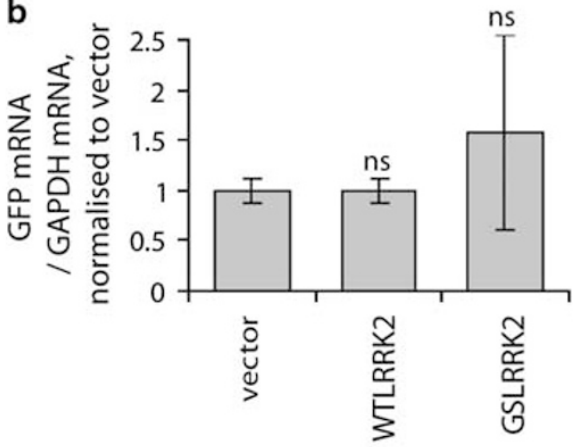

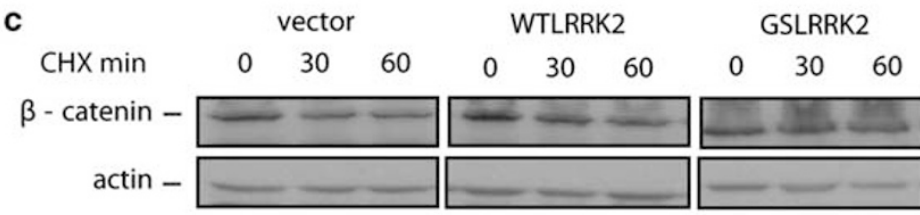

d

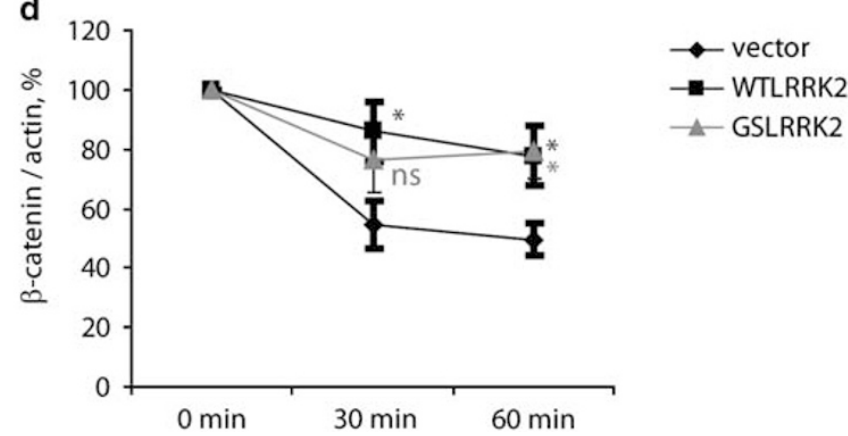

Figure 3 Increased protein levels due to LRRK2 overexpression are not a function of transcription or translation. (a) qRT-PCR analysis of $m R N A$ levels of overexpressed GFP-tagged $\alpha$-synuclein and (b) mRNA levels of overexpressed GFP in HeLa cells transfected with WTLRRK2, GSLRRK2 or vector. mRNA levels are normalised to GAPDH. Graphs show data from two independent experiments performed in triplicate. Data are shown as means (normalised to vector-transfected cells) \pm S.D. of replicates. Statistical analysis was performed on raw replicate data within each experiment by two-tailed, unpaired Student's $t$-test and representative $P$-values are shown. ns $=$ not significant, ${ }^{\star} P<0.05,{ }^{* \star} P<0.01, N=2$. (c) Degradation of $\beta$-catenin is slowed in LRRK2-transfected cells. HeLa cells were transfected with LRRK2 or control DNA. After $48 \mathrm{~h}$ of transfection, protein synthesis was inhibited by treatment with $\mathrm{CHX}$ for the indicated time (minutes). Degradation of $\beta$-catenin was assessed by western blot. (d) Quantification of (c). Graph shows data from three independent experiments as means (normalised to vector-transfected cells at time point zero) \pm S.E.M. Statistical analysis was performed on normalised data by two-tailed, paired Student's $t$-test. ns $=$ not significant, ${ }^{\star} P<0.05, N=3$

gain-of-function mechanism, we studied the effects of LRRK2 overexpression.

HeLa cells were transfected with either wild-type (WT) or A53T-mutant GFP-tagged $\alpha$-synuclein (which causes rare forms of autosomal dominant PD). Simultaneously, cells were transfected with wild-type (WTLRRK2) or G2019S-mutant (GSLRRK2) Flag-tagged LRRK2, or vector DNA as a control. The G2019S mutation in LRRK2 is the most common cause of hereditary PD and is considered toxic due to excessive kinase activity. ${ }^{12}$ As an additional control, we included httQ23, the non-pathogenic exon 1 of wild-type huntingtin protein containing 23 glutamine repeats, which was used to control for possible effects due to exogenous protein expression. Both WTLRRK2 and GSLRRK2 overexpression led to increased levels of WT and A53T-mutated overexpressed $\alpha$-synuclein (Figures 1a-d).

Besides an increase in $\alpha$-synuclein levels, accumulation of ubiquitinated proteins is also described in LRRK2 PD. ${ }^{3}$ Interestingly, we observed that overexpression of both WTLRRK2 and GSLRRK2 increased the ubiquitinated proteins in HeLa cells and mouse primary cortical neurons (Figures 1e-g and Supplementary Figure S1). We studied cortical neurons, as LRRK2 is significantly expressed in this region, where inclusion pathology has been observed. ${ }^{3}$ As previously described, overexpression of LRRK2 resulted in the formation of LRRK2 aggregates. ${ }^{13,14}$ However, these rarely co-localised with ubiquitin inclusions (Figure $1 \mathrm{f}$ and Supplementary Figure S1), suggesting that the observed increase in ubiquitin inclusion formation was not caused by the sequestration of ubiquitin in LRRK2 aggregates.

LRRK2 affects $\alpha$-synuclein and other proteins. To test if LRRK2 had more general effects on protein homoeostasis, we tested whether its overexpression had an effect on another protein that aggregates intracellularly. Mutant polyglutamine-expanded huntingtin fragment (httQ74) is an aggregate-prone protein that rapidly forms inclusions after transfection in cells and leads to cell death. In this study, we have used the mutated polyglutamine protein as an exogenous 'reporter' for protein homoeostasis. LRRK2 


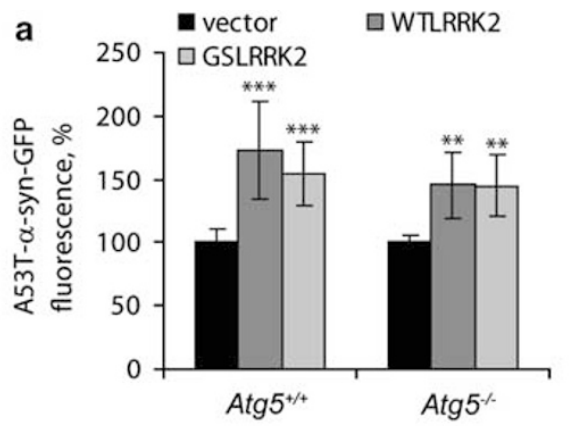

b
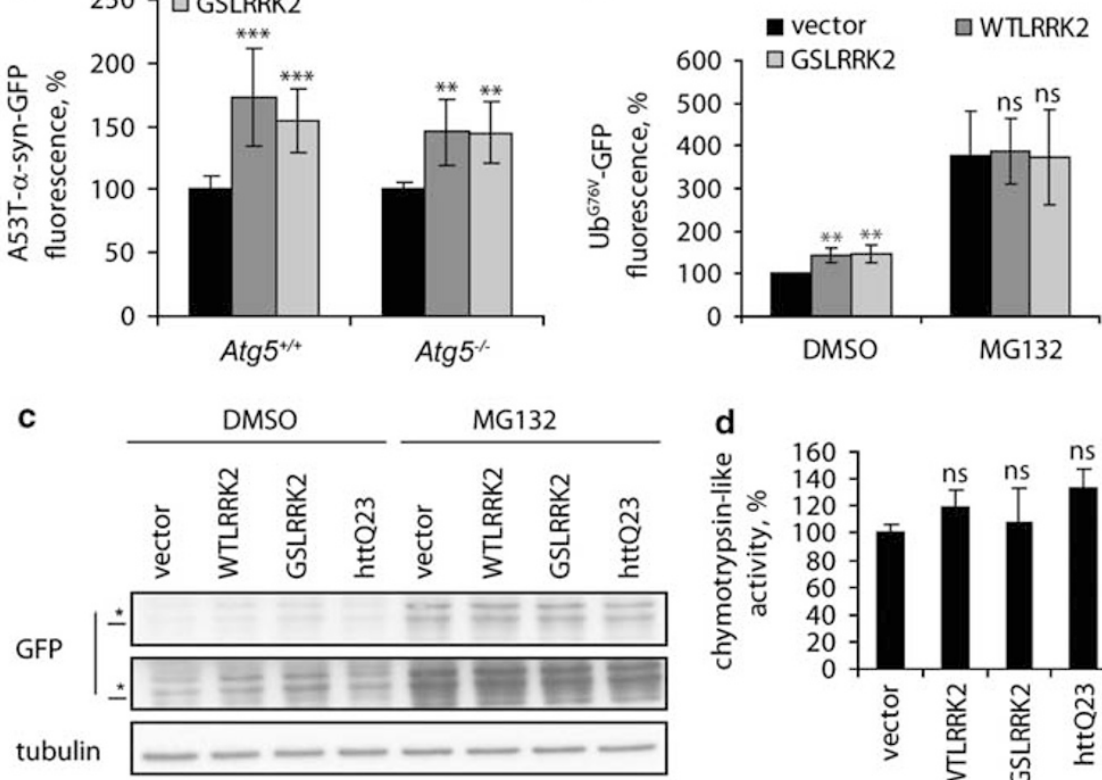

e

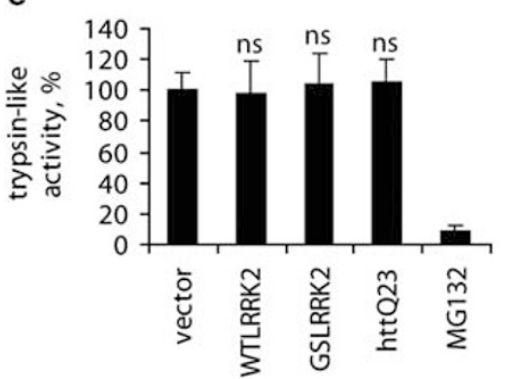

f

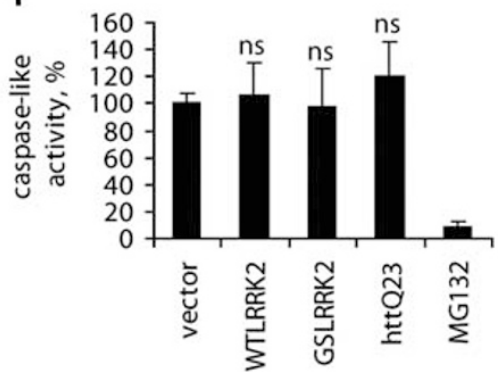

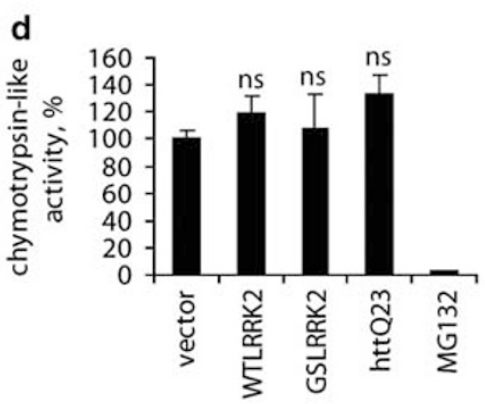

Figure 4 LRRK2 overexpression interferes with the UPS but not with proteasome catalytic activity. (a) Increased levels of GFP-tagged A53T- $\alpha$-synuclein in Atg $5^{+/+}$and Atg $5^{/-}$MEFs in response to LRRK2 overexpression. Atg $5^{+/+}$and Atg $5^{-/}$MEFs were transfected with WTLRRK2, GSLRRK2 or empty vector and co-transfected with GFPtagged A53T- $\alpha$-synuclein. GFP fluorescence was measured $48 \mathrm{~h}$ after transfection by flow cytometry and normalised to vector controls. Graph shows data from four independent experiments performed in triplicates as normalised means \pm S.D. of replicates. Statistical analysis was performed on raw replicate data of each individual experiment by two-tailed, unpaired Student's $t$-test and representative $P$-values are shown. ns $=$ not significant, ${ }^{\star \star} P<0.01,{ }^{\star \star \star} P<0.001, N=4$. (b) The UPS-specific reporter $\mathrm{Ub}^{\mathrm{G} 76 \mathrm{~V}}$-GFP was overexpressed in HeLa cells along with LRRK2 or vector. Immediately after transfection, cells were treated with the proteasome inhibitor MG132 (10 $\mu \mathrm{M}$ ) or vehicle (DMSO), and GFP fluorescence was measured $12 \mathrm{~h}$ later by flow cytometry. Increased levels of fluorescence were measured in LRRK2-transfected, vehicle-treated cells, compared with vector-transfected, vehicle-treated cells. Treatment with MG132 increased levels of fluorescence in all samples, and no difference was detected between LRRK2- and vector-transfected cells in the presence of MG132. Graph shows data from three independent experiments performed in triplicates (normalised to vectortransfected, DMSO-treated cells) as means \pm S.D. of replicates. Statistical analysis was performed on raw replicate data of each individual experiment by two-tailed, unpaired Student's t-test and representative $P$-values are shown. ns $=$ not significant, ${ }^{* \star} P<0.01, N=3$. (c) HeLa cells were transfected and treated as in (b). Protein levels were measured by western blotting $12 \mathrm{~h}$ after transfection. Increased Ub ${ }^{\mathrm{G} 6 \mathrm{~V}}$ _GFP protein levels are measured in response to LRRK2 overexpression in vehicle-treated, but not in MG132-treated cells, compared with the respective vector control. Blot shown is representative for four independent experiments. Asterisk (*) indicates unspecific band. (d-f) Proteasome catalytic activities in HeLa cell lysates transfected with GFP and LRRK2, httQ23 or vector. GFP-positive cells were sorted $48 \mathrm{~h}$ post-transfection and proteasome activity was analysed by a fluorigenic in vitro assay. As a control, proteasome activities were also measured in vector-transfected lysates treated with MG132. Data from three independent experiments are shown as means (normalised to vector-transfected cells) \pm S.D. of replicates. Statistical analysis was performed on raw replicate data by twotailed, unpaired Student's $t$-test and representative $P$-values are shown. ns = not significant, $N=3$. (g) Cell lysates from the same conditions as in a-c were immunoblotted with antibodies against the indicated proteasome subunits. Blots are representative for three independent experiments $(N=3)$. See also Supplementary Figure $S 4$

overexpression increased the percentage of transfected COS-7 cells with httQ74GFP aggregates and cell death (Figures $2 a$ and $b$ ). To assess the specificity of this effect, we also tested the effect of LRRK1 on httQ74 aggregation. Overexpression of LRRK1, a paralogue of LRRK2 that has a very similar domain structure, ${ }^{15}$ did not affect httQ74 aggregation in COS-7 cells (Supplementary Figure S2A), suggesting that this effect is specific to PD-associated LRRK2.
Importantly, LRRK2 aggregates do not co-localise with httQ74 aggregates (Supplementary Figure S2B), suggesting that LRRK2 does not sequester httQ74 but promotes its aggregation by other means. Such an increase in the number of aggregate-containing cells can occur either because of a specific increase in aggregation or due to other factors, as httQ74 aggregation is concentration-dependent. ${ }^{16}$

To further assess the specificity of the LRRK2 effect on protein homoeostasis, we measured levels of GFP in cells 
overexpressing LRRK2 and found this to be increased (Figure 2c). As the aggregate-prone protein httQ74 did not increase GFP fluorescence (Supplementary Figure S2C), this suggests that the increase in GFP fluorescence caused by the overexpression of LRRK2 is not a generic effect of aggregateprone proteins, and may indicate that GFP and other proteins are not simply sequestered in LRRK2 aggregates. LRRK2 overexpression also caused an increase in GFP fluorescence in a neuroblastoma cell line, while LRRK1 did not affect GFP fluorescence, suggesting that the observed effect on protein levels is specific for the PD-associated LRRK2 (Figures 2d and e).

We also tested the effect of LRRK2 on levels of an endogenous protein. As shown in Figure 2f, levels of p53 (a well-characterised proteasome substrate ${ }^{17}$ ) were elevated in
LRRK2-transfected cells. Together, these results suggest that the effect of LRRK2 is not only specific for overexpressed $\alpha$-synuclein but can also cause the accumulation of endogenous as well as other exogenous intracytosolic proteins.

LRRK2 acts independently of transcription or translation. It has been previously described that WTLRRK2 overexpression can increase WT- $\alpha$-synuclein mRNA and protein levels through ERK pathway activation in HEK293 cells. ${ }^{18}$ However, we did not observe ERK activation in our cells (Supplementary Figure S3). Furthermore, we confirmed that LRRK2 overexpression did not affect transcription of GFP-tagged $\alpha$-synuclein or GFP, suggesting that the increased protein levels due to LRRK2 overexpression is not caused by increased protein synthesis (Figures $3 a$ and b).
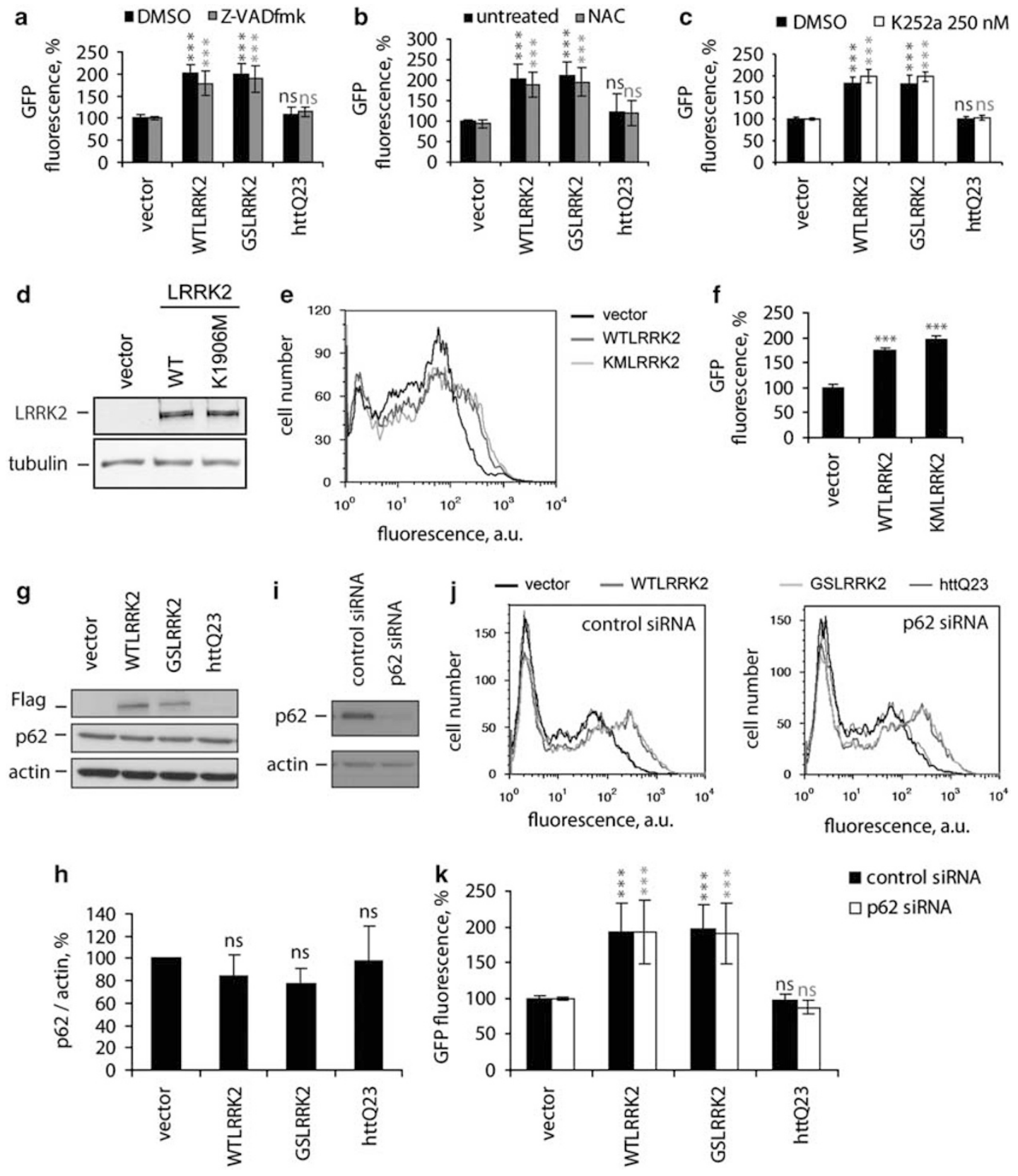

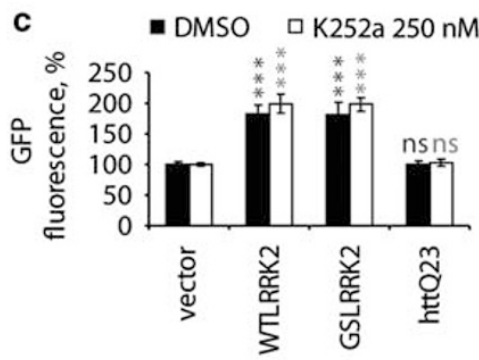

f

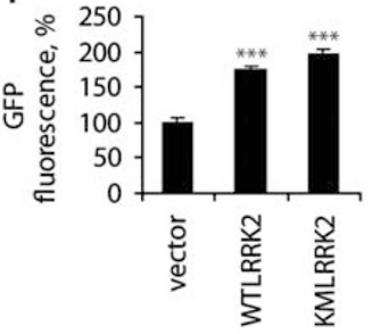

- control siRNA

p 62 siRNA 
We next determined whether the effects of LRRK2 on protein levels were dependant on translation. In cells transfected with empty vector, levels of the proteasome substrate $\beta$-catenin decreased over $60 \mathrm{~min}$ of treatment with cycloheximide due to blocked translation and continuing degradation. $\beta$-catenin levels decayed more slowly in cells transfected with LRRK2, suggesting that it increases protein levels via impairment of degradation rather than upregulation of protein synthesis (Figures $3 c$ and d).

Expression of LRRK2 interferes with the UPS. The proteins that we have so far shown to be affected by LRRK2 overexpression can be degraded by both the UPS and macroautophagy, although with different preferences (Supplementary Figure S4A). We next investigated the effect of LRRK2 overexpression on levels of A53T- $\alpha$-synuclein and GFP in $A t g 5^{-}$autophagy- incompetent and matched-wild-type mouse embryonic fibroblasts (MEFs, ${ }^{19}$ ). A53T- $\alpha$-synuclein and GFP were increased in these matched cell lines (Figures $4 a$ and $b$ ), suggesting that the protein accumulation due to LRRK2 overexpression was autophagy independent.

We next aimed to assess the role of the UPS in LRRK2mediated protein accumulation. Proteins are targeted for proteasomal degradation after they are tagged with ubiquitin chains, and we used an artificial proteasome substrate where ubiquitin is N-terminally fused to GFP (Ub G76V_GFP). ${ }^{20}$ Under normal conditions, this modified GFP is quickly degraded and hardly detectable. However, impaired UPS function can be sensitively detected by increased levels of this reporter. ${ }^{17,20}$ LRRK2 overexpression caused increased Ub ${ }^{\mathrm{G} 76 \mathrm{~V}}$-GFP levels (Figures $4 \mathrm{~b}$ and c, Supplementary Figure S4C) without affecting transcription (Supplementary Figure S4D), suggesting that LRRK2 interferes with Ub ${ }^{G 76 V}$-GFP degradation by the UPS. Importantly, proteasome inhibition with MG132 led to a similar accumulation of $\mathrm{Ub}^{\mathrm{G} 76 \mathrm{~V}}$-GFP fluorescence
(Figure 4b) and protein (Figure 4c) in LRRK2- and controltransfected cells, suggesting that LRRK2 overexpression affects protein homoeostasis by impairing the UPS.

We further tested the role of the proteasome by performing in vitro assays in cell lysates to measure the three catalytic activities of the proteasome active sites. While all three catalytic activities were unaffected by LRRK2 overexpression (Figures $4 d-f$ ), it is important to note that this assay is independent of upstream processes like ubiquitination and deubiquitination.

We further showed that expression levels of various proteasome subunits were unaffected by LRRK2 overexpression (Figure 4g). Together, these results suggest that LRRK2 overexpression causes protein accumulation via UPS impairment, but does not affect the protein levels or the catalytic activities of the proteasome itself.

Effect of LRRK2 on protein levels is independent of its toxicity. It has been previously shown that mutant LRRK2 is toxic when transfected into neurons or neuroblastoma cell lines, leading to neurite process reduction and apoptosis. ${ }^{13,14,21}$ We therefore investigated whether cell death caused by LRRK2 overexpression affected protein accumulation. We confirmed a cytotoxic effect of LRRK2 overexpression on HeLa cells compared with LRRK1 and httQ23 (Supplementary Figures S5A and B). As previously shown, cell death due to LRRK2 overexpression coincided in most cells with caspase 3 activation (Supplementary Figures S5B and C). ${ }^{21}$ Indeed, the caspase inhibitor Z-VADfmk reduced the activation of caspase 3 caused by LRRK2 overexpression, and this coincided with reduced cell death ${ }^{21}$ (Supplementary Figures S5A-C). The accumulation of GFP fluorescence due to LRRK2 overexpression was, however, not diminished in the presence of antiapoptotic and antioxidant (NAC) drugs (Figures $5 \mathrm{a}$ and b). Together, the

Figure 5 The increase in protein levels is not due to oxidative stress, increased cell death, kinase activity or p62. (a) HeLa cells were transfected with empty vector, WTLRRK2, GSLRRK2 or httQ23, and co-transfected with GFP as a reporter protein. Cells were treated immediately after transfection with the caspase inhibitor Z-VADfmk and GFP fluorescence intensity was measured by flow cytometry $48 \mathrm{~h}$ after transfection. The increase in GFP fluorescence in response to LRRK2 overexpression was not prevented by caspase inhibition. (b) Cells were transfected as in (a) and treated immediately after transfection with the antioxidant $N$-acetyl-L-cysteine (NAC). Cellular GFP fluorescence was measured $48 \mathrm{~h}$ later by flow cytometry. The increase in fluorescence due to LRRK2 overexpression was not prevented by treatment with NAC. (c) HeLa cells were transfected as in (a) and treated with $250 \mathrm{nM} \mathrm{K252a}$ or DMSO for $48 \mathrm{~h}$. Cellular GFP fluorescence was measured $48 \mathrm{~h}$ later by flow cytometry. The increase in fluorescence due to LRRK2 overexpression was not prevented by treatment with the LRRK2 kinase inhibitor. (a-c) All graphs show means of normalised data \pm S.D. of replicates from three independent experiments. Statistical analysis was performed on raw replicate data by two-tailed, unpaired Student's $t$-test and representative $P$-values are shown. ns $=$ not significant, ${ }^{* \star \star} P<0.001, N=3$; black symbols reflect $P$-values compared with DMSO-treated cells, grey symbols reflect $P$-values compared with drugtreated cells. Note, that data shown in graph 6D for untreated cells are the same data as in Supplementary Figure S2B, where httQ74 was included. (d) HeLa cells were transfected with WTLRRK2 or KMLRRK2 or vector DNA, and cell lysates were analysed $48 \mathrm{~h}$ after transfection by western blotting. Similar levels of WTLRRK2 and KMLRRK2 protein were detected. (e and f) HeLa cells were transfected as in (d), and co-transfected with GFP. GFP fluorescence was measured $48 \mathrm{~h}$ after transfection by flow cytometry. (e) Increased levels of GFP fluorescence were detected in both WTLRRK2- and KMLRRK2-transfected cells (dark grey and light grey line, respectively), compared with vectortransfected cells (black line). (f) Graph shows data normalised to vector-transfected cells for three independent experiments performed in triplicates as means \pm S.D. of replicates. Statistical analysis was performed on raw replicate data by two-tailed, unpaired Student's $t$-test and representative $P$-values are shown. ${ }^{* \star \star} P<0.001, N=3$. (g) HeLa cells transfected with GFP and DsRed were co-transfected with LRRK2 or control DNA. p62 protein levels were assessed by western blotting $48 \mathrm{~h}$ later following FACS sorting for double-positive cells and a recovery period of $24 \mathrm{~h}$. Blot is the same blot as shown in Figure $4 \mathrm{~g}$ and representative for three independent experiments. (h) Quantification of $(\mathbf{g})$. Data normalised to vector-transfected cells are shown for three independent experiments as means \pm S.D. Statistical analysis was performed on raw data by two-tailed, paired Student's $t$-test. $\mathrm{ns}=$ not significant, $N=3$. (i) Knockdown of p62. HeLa cells were twice transfected with $50 \mathrm{nM}$ siRNA targeted against $\mathrm{p} 62$. p62 levels were assessed by western blot $48 \mathrm{~h}$ after the second knockdown. (j and $\mathbf{k}$ ) HeLa cells were transfected with siRNA targeted against p62 as in (i). GFP and LRRK2 or control DNA was co-transfected at the second knockdown. GFP fluorescence was measured by flow cytometry $48 \mathrm{~h}$ after the second transfection. (j) Representative flow cytometry histograms show fluorescence intensity plotted against cell number. Left: in the presence of non-targeting control siRNA, increased fluorescence is observed in response to WTLRRK2 and GSLRRK2 overexpression (dark grey and light grey line, respectively), compared with vector- or httQ23-transfected cells (thick and thin black line, respectively). Right: in the presence of p62-targeting siRNA, the same increase in cellular fluorescence as with control siRNA is observed in LRRK2-transfected cells. (k) Graph shows data (normalised to vector-transfected cells) from four independent experiments performed in triplicates as means \pm S.D. Statistical analysis was performed on raw replicate data by two-tailed, unpaired Student's $t$-test and representative $P$-values are shown. ns $=$ not significant, ${ }^{* \star \star} P<0.001, N=4$ 
above data suggest that the accumulation of various proteins after LRRK2 overexpression is not a consequence of caspase activation or reactive oxygen species.

LRRK2 effect on protein levels is independent of its kinase activity. Some studies investigating LRRK2 toxicity revealed a critical role for the kinase domain, supporting a gain-of-function effect due to the the increased kinase activity. ${ }^{12,13,22}$ We therefore investigated whether the kinase activity of LRRK2 is relevant for its observed effect on protein accumulation. As shown in Figure $5 \mathrm{c}$ and Supplementary Figure S5D, the increase in GFP levels caused by LRRK2 overexpression was not reduced by two published LRRK2 kinase inhibitors. ${ }^{23,24}$ Furthermore, the artificial kinase-dead mutation construct K1906M (KMLRRK2) $^{10}$ also increased GFP fluorescence similarly to WTLRRK2 (Figures $5 d-f$ ). In conclusion, the effect of LRRK2 on protein levels seems to occur independently of the kinase activity.
Accumulation of proteins upon LRRK2 expression is independent of p62. We have recently shown that overexpression of the ubiquitin-binding protein, p62, inhibits degradation of substrates destined for the proteasome at a step upstream of proteasome catalytical activity, ${ }^{17}$ resulting in a very similar phenotype to that described in this study. In the present study LRRK2 overexpression itself did not affect endogenous levels of p62 (Figures $5 \mathrm{~g}$ and $\mathrm{h}$ ). In order to test if the effect of LRRK2 on the UPS was p62 dependent, we reduced p62 levels by transfecting p62 siRNA into HeLa cells (Figure 5i), and tested the effect of LRRK2 overexpression on levels of GFP as before by flow cytometry. In the presence of control siRNA, LRRK2 led to an increase of GFP fluorescence as described before (Figure 2). This increase was not affected by siRNA directed against p62 (Figures 5j and k), suggesting that p62 is not involved in the impairment of the UPS by LRRK2.

Co-expression of HSP70 enhances the effect of LRRK2 on protein accumulation. HSP70 is a chaperone that
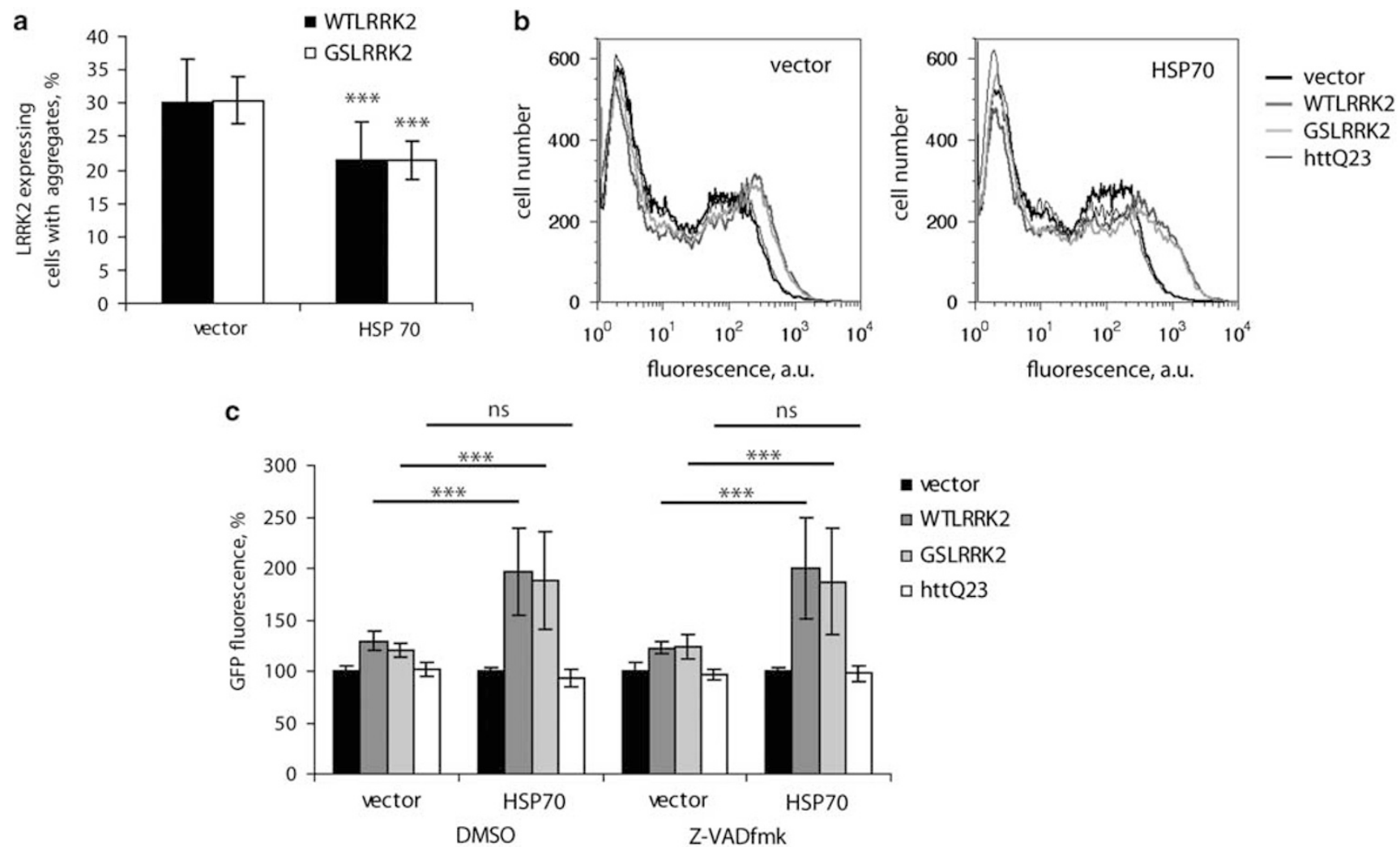

Figure 6 The increase in protein levels can be modulated by HSP70. (a) COS-7 cells transfected with WTLRRK2 or GSLRRK2 were co-transfected with either vector or HSP70 in a molar ratio of 1:2 (LRRK2/HSP70). Cells were fixed $24 \mathrm{~h}$ post-transfection, stained with antiFlag antibody, and LRRK2 aggregates were scored in at least 200 cells per replicate. Graph shows mean percentage of cells containing LRRK2 aggregates \pm S.E.M. from three independent experiments. Statistical analysis was performed by unconditional logistical regression analysis. ${ }^{\star \star} P<0.001, N=3$. (b and $\mathbf{c}$ ) HSP70 co-expression enhances the accumulation of GFP protein in response to LRRK2 overexpression. HeLa cells were transfected with WTLRRK2, GSLRRK2, vector or httQ23 and co-transfected with GFP. Additionally, cells were transfected with vector or HSP70. Molar ratio of LRRK2/HSP70 is 1:2. Cells were treated immediately after transfection with DMSO or Z-VADfmk. GFP fluorescence was measured $48 \mathrm{~h}$ posttransfection by flow cytometry. (b) Representative flow cytometry histograms show increased cellular GFP fluorescence in response to WTLRRK2 and GSLRRK2 overexpression (dark and light grey line, respectively), compared with cells transfected with vector or httQ23 (thick and thin black line, respectively). This increase in fluorescence is enhanced when HSP70 is co-overexpressed (right histogram). (c) Quantification of (b). Graph shows data (normalised to vector-transfected cells) from three independent experiments performed in triplicates as mean \pm S.D. of replicates. Statistical analysis was performed by two-tailed, unpaired Student's $t$-test on replicates of individual experiments and representative $P$-values are shown. $n s=$ not significant, ${ }^{* \star \star} P<0.001, N=3$ 
assists folding of misfolded proteins, and LRRK2 has been described as an aggregating protein. Both WTLRRK2 and GSLRRK2 aggregation are decreased by overexpressed HSP70 (Figure 6a). Accordingly, we tested whether coexpression of HSP70 affected the LRRK2-mediated accumulation of GFP. We overexpressed LRRK2 along with GFP and either control DNA or HSP70 and measured GFP fluorescence. HSP70 overexpression enhanced the GFP increase mediated by LRRK2 overexpression (Figures $6 \mathrm{~b}$ and $\mathrm{c}$ ). This was also seen in the presence of the antiapoptotic drug Z-VADfmk (Figures $6 \mathrm{~b}$ and $\mathrm{c}$ ), suggesting that HSP70 acts on LRRK2-mediated protein accumulation through a mechanism other than its anti-apoptotic effect. ${ }^{25}$

Interestingly, HSP70 overexpression had no significant effect on soluble LRRK2 levels (Supplementary Figures S6A and B). As HSP70 decreases LRRK2 aggregation and enhances its effect on protein levels, the accumulation of proteins caused by LRRK2 is not simply due to its aggregation. Instead, our data suggest that LRRK2 is more accessible in the presence of HSP70, for example, as mono- or oligomeric forms, and that these species may mediate the accumulation of other proteins.

LRRK2 affects protein accumulation in vivo. To confirm the in vivo relevance of our observations, we performed transient DNA overexpression experiments in zebrafish (Figure 7a). Transient co-expression of WTLRRK2 or GSLRRK2 with Ub ${ }^{\text {G76V }}$-GFP caused a significant increase in the number of embryos with mosaic GFP expression, compared with co-expression of $\mathrm{Ub}^{\mathrm{G} 76 \mathrm{~V}}$-GFP with LRRK1 or the vector control constructs (Figures $7 b$ and $c$ ), which we confirmed by western blot (Figure 7d). Together, these results demonstrate that clearance of transiently expressed $\mathrm{Ub}^{\mathrm{G} 76 \mathrm{~V}}$-GFP is impaired when co-expressed with WTLRRK2
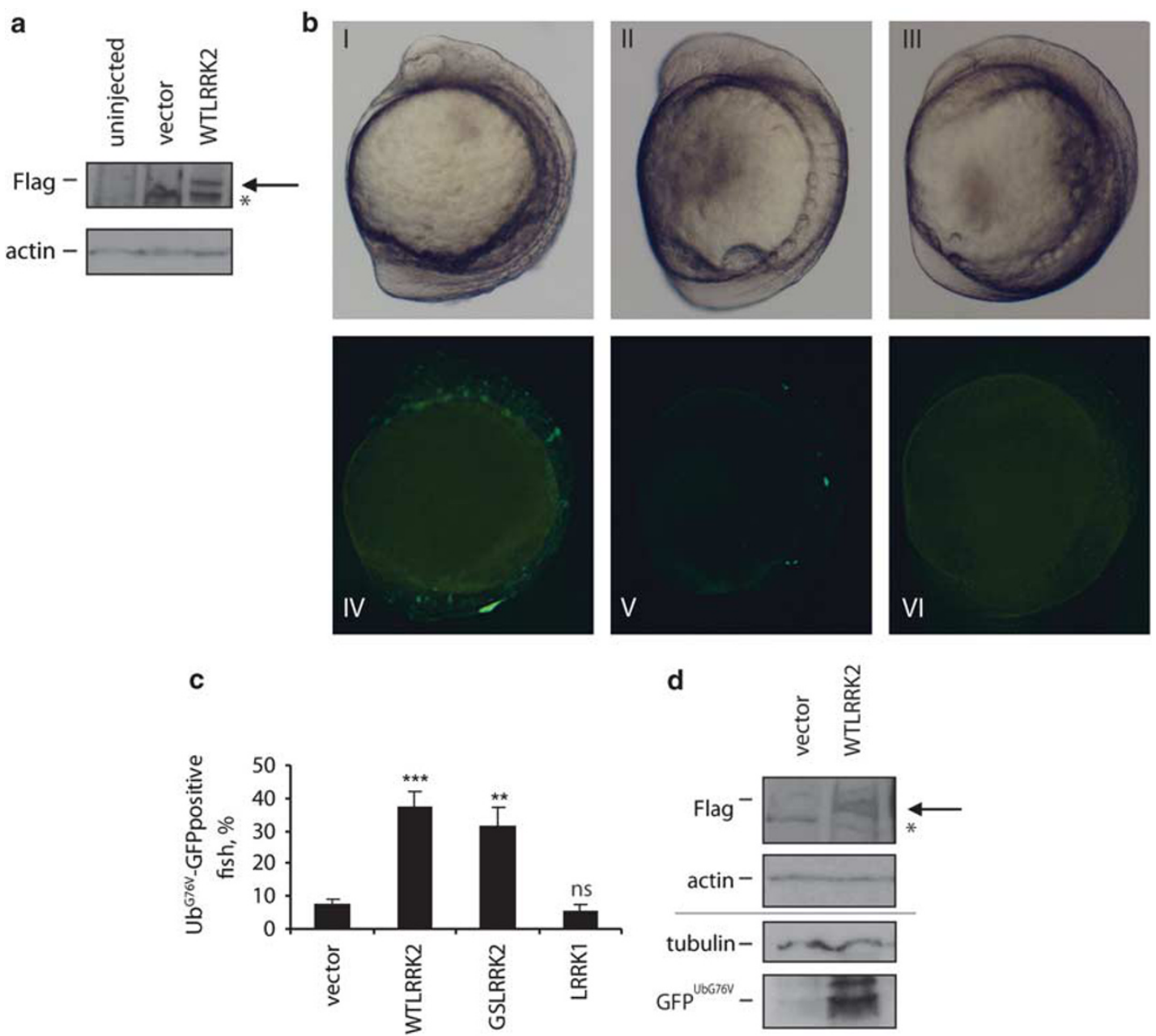

Figure 7 LRRK2 expression causes UPS disruption in zebrafish. (a) LRRK2 expression in zebrafish. Zebrafish embryos were injected with empty vector or WTLRRK2 at the 1-2 cell stage and lysed at 12 h.p.f. LRRK2 protein was detected by western blotting. Asterisk indicates unspecific band; arrow indicates LRRK2 band. (b) Zebrafish embryos at 12 h.p.f. following injection of Ub ${ }^{\text {G76V }}$-GFP with either WTLRRK2 (I \& IV) or empty vector (II \& V). Uninjected siblings (III \& VI) are included for comparison. Injection of WTLRRK2 (IV) or GSLRRK2 (not shown) resulted in a high and stable level of Ub ${ }^{G 76 V}$-GFP expression. Typically, expression was observed in more than 30 cells. Injection of LRRK1 (not shown) or vector (V) resulted in a low level of Ub ${ }^{\mathrm{G} 76 \mathrm{~V}}$-GFP expression. In vector- and LRRK1-injected embryos, expression was observed in less than 10 cells

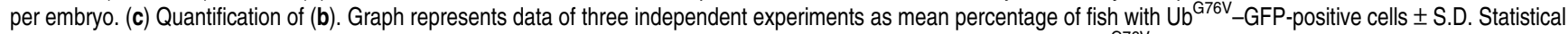
analysis was performed by two-tailed, unpaired Student's $t$-test. ${ }^{\star *} P<0.01,{ }^{* \star *} P<0.001, N=3$. (d) Increased levels of Ub ${ }^{\text {G76V }}$-GFP were detected in WTLRRK2-expressing zebrafish by western blot. Asterisk $\left(^{*}\right)$ indicates unspecific band; arrow indicates LRRK2 band 
or GSLRRK2, suggesting that overexpression of LRRK2 causes disruption of the UPS in vivo.

\section{Discussion}

PD patients with LRRK2 mutations have pleiomorphic pathology, where $\alpha$-synuclein and/or tau and/or ubiquitin have been detected in intraneuronal aggregates. ${ }^{3}$ Furthermore, the phenotype of LRRK2 PD is very similar to the idiopathic PD. ${ }^{3,5}$ Accordingly, it is believed that LRRK2 acts far upstream in the unknown pathway leading to the development of the disease, and that deciphering the roles of LRRK2 will provide significant insight into PD pathogenesis of PD.

The abnormal accumulation of cytoplasmic inclusions with ubiquitin is a common feature in $\mathrm{PD}$ and other neurodegenerative diseases. This could arise if an interference with protein degradation was one of the principal mechanisms leading to the development of the diseases. Our data demonstrate the involvement of LRRK2 in protein homoeostasis. We have provided a mechanism for the accumulation of multiple proteins due to LRRK2 overexpression by showing that this results in impaired flux through the UPS. This would result in the accumulation $\alpha$-synuclein, tau and ubiquitin in PD patients with LRRK2 mutations, if the disease is due to excess LRRK2 function, as has been proposed. $^{7,8}$ Our data are further supported by the observation that LRRK2 overexpression enhances ubiquitin accumulation in mice. ${ }^{7}$

Our data suggest that UPS malfunction is the major cause behind the accumulation of proteins in this context, as LRRK2 overexpression resulted in no additional protein reporter protein accumulation in proteasome-inhibited cells (Figure 4). Furthermore, LRRK2 overexpression led to protein accumulation in autophagy-incompetent cells (Figure 4), suggesting that the effects are autophagy-independent. The idea of UPS impairment by LRRK2 is further supported by the accumulation of a specific proteasome substrate in both LRRK2expressing cells and zebrafish (Figure 7).

We have shown that LRRK2 did not affect the catalytic activity of the proteasome or expression levels of proteasomal core subcomplexes (Figure 4). Moreover, co-expression of HSP 70 decreased the tendency of LRRK2 to form aggregates and at the same time enhanced LRRK2-mediated protein accumulation (Figure 6). Together, these findings suggest that accumulation of proteins after LRRK2 expression is not caused by sequestration of these proteins or proteasomal subunits into LRRK2 aggregates, but rather by mono- or oligomeric forms of LRRK2 acting on the UPS. It is not clear at which level the UPS is perturbed by LRRK2. The cascade of reactions acting upstream of the proteasome catalytic activity is complex and finely tuned. It is possible that ubiquitination or de-ubiquitination may be perturbed or that recognition or transport of ubiquitinated proteins to the proteasome may be altered.

Disease-causing LRRK2 mutations are found throughout the protein. Although increased kinase activity was consistently shown for the most prominent G2019S mutation, data for other mutations are contradictory. ${ }^{11,26}$ Thus, mutant LRRK2 may confer pathogenic effects through other functions. In our experimental conditions, the wild-type form of LRRK2 had a significant effect on reporter protein levels and ubiquitin levels that were similar to G2019S-mutated LRRK2. Also, chemical or genetic inhibition of LRRK2 kinase activity did not modulate the accumulation of proteins, suggesting that LRRK2 kinase activity is not a major determinant of its UPS-inhibitory phenotype. This is supported by in vivo data. ${ }^{27}$

Mutations in LRRK2 cause PD through a gain-of-function mechanism, and we therefore overexpressed the protein in order to mimic a gain-of-function. Although the kinase activity does not seem to be critical for the impairment of the UPS system, it is surprising that the G2019S mutation does not confer additional toxicity compared with WTLRRK2. It is feasible that a kinase-independent toxic mechanism depends on exceeding a threshold level of activity, which may be lowered by pathogenic mutations. This kind of mechanism is supported by the finding that homo- and heterozygous carriers of LRRK2 mutations are clinically indistinguishable. ${ }^{28}$ If we assume that LRRK2 function needs to exceed a threshold level in order to become toxic, then this may explain why we could not observe a difference in the effect of WTLRRK2 and GSLRRK2 on the impairment of the UPS in an overexpression study.

Alternatively, we may have missed a small but biologically significant difference in effect. The overexpression of LRRK2 may result in a saturated environment where target proteins or auxiliary factors become rate limiting and therefore reduce the true effect size. Even if these other proteins are in abundance, overexpression might over-represent the impact of the protein to such an extent that small differences are not detectable. However, even a small difference may have deleterious consequences over the course of a lifetime, as in PD.

Lastly, we cannot exclude the possibility that UPS inhibition is a wild-type function of LRRK2 unrelated to PD. Although we do not consider this a likely scenario, we believe the involvement of LRRK2 with the UPS as relevant, as it provides some insights into LRRK2's function, which is poorly understood. It is also critical to understand the consequences of LRRK2 overexpression for understanding models of LRRK2 PD, as all Drosophila, ${ }^{29-31}$ mouse $^{7,32,33}$ and cellbased $^{9,12,13,21,22,34-36}$ models published to date have relied on overexpression.

Altogether our data support a role for LRRK2 in the UPS pathway. It is possible that the accumulation of UPS substrates in LRRK2-mediated PD may be caused by a similar mechanism to LRRK2 overexpression, consistent with a gain-of-function model. A better knowledge of the function of LRRK2 will assist further generation of cellular and animal models and allow for the development of rational therapeutic strategies.

\section{Materials and Methods}

Plasmids. Flag-tagged wild-type LRRK2 (WTLRRK2) and G2019S mutant (GSLRRK2) were kindly provided by CA Ross, ${ }^{37}$ the K1906M LRRK2 mutant was generated from a myc-tagged wild-type LRRK2 construct kindly provided by M Cookson. ${ }^{36}$ The LRRK1 construct was kindly provided by D Korr. ${ }^{15} \mathrm{HD}$ gene exon 1 fragment with 74 or 23 polyQ repeats in pEGFP-C1 (Clontech, Palo Alto, CA, USA) or in pHM6 (Roche Diagnostics, Mannheim, Germany) and pEGFP- $\alpha$-synuclein A53T or wild-type $\alpha$-synuclein in pEGFP-C1 were described previously. ${ }^{16,38}$ Plasmid pEGFP-Ub ${ }^{\mathrm{G} 76 \mathrm{~V}}$ (Ub $\left.{ }^{\mathrm{G} 76 \mathrm{~V}}-\mathrm{GFP}\right)$ was kindly provided by N Dantuma. ${ }^{20}$ DsRed-C1 (DsRed) was from Clontech and pCDNA3.1 was from Invitrogen (Carlsbad, CA, USA). 
Cell culture, transfections and flow cytometry analysis. HeLa, SHSY5Y and COS-7 cells were grown in DMEM medium (Sigma, St. Louis, MO, USA) supplemented with $10 \%$ foetal calf serum, penicillin/streptomycin and $2 \mathrm{mM} \mathrm{L-}$ glutamine at $37^{\circ} \mathrm{C}$ in $5 \% \mathrm{CO}_{2}$. This medium was supplemented with $5 \mathrm{mg} / \mathrm{ml}$ geneticin (Sigma) for the culture of the Ub ${ }^{\mathrm{G} 76 \mathrm{~V}}$-GFP-expressing stable cell line (kind gift from NP Dantuma). PC12 cells were grown in DMEM medium supplemented with $10 \%$ horse serum, $5 \%$ foetal calf serum (Sigma), penicillin/streptomycin (Sigma) and $2 \mathrm{mM} \mathrm{L-glutamine} \mathrm{(Sigma)} \mathrm{at} 37^{\circ} \mathrm{C}$ in $10 \% \mathrm{CO}_{2}$. PC12 cells were differentiated by the addition of $1 \mathrm{mM}$ NGF (Upstate, Milton Keynes, UK) for 5 days before transfection. Transfections were performed using Lipofectamine Plus or Lipofectamine 2000 (Invitrogen) according to the manufacturer's instructions.

For primary neuron cultures, cortex was dissected from E18 mouse embryo brains in DMEM containing pyruvate, $4.5 \mathrm{~g} / \mathrm{l}$ glucose and L-glutamine. The tissue was triturated to a single-cell suspension using a 200- $\mu$ l pipette until no pieces could be seen. Cells were then plated at the desired density on poly-D-lysine-coated coverslips in DMEM containing 2\% B-27 (Invitrogen), 1\% PSF and 1\% foetal calf serum. The cells were transfected with Lipofectamine 2000 (Invitrogen) according to the manufacturer's instructions.

For flow cytometry cells were transfected with a GFP-containing reporter protein and analysed with FACS Calibur $48 \mathrm{~h}$ after transfection. Data represent the geometric mean of green intensity.

For cell sorting experiments, HeLa cells were transfected with both GFP and DsRed, and either control or WTLRRK2 or GSLRRK2 DNA in a molar ration of $1: 10$. Cells were sorted $48 \mathrm{~h}$ after transfection for GFP and DsRed double-positive cells based on the assumption that double-transfected cells are more likely to be also transfected with LRRK2 or control DNA than cells that were not or only single transfected. We thus isolated cell populations that were enriched in tripletransfected cells, expressing GFP and DsRed and either httQ23, WTLRRK2 or GSLRRK2. These cells were grown for an additional $24 \mathrm{~h}$ in order to recover from the sorting process. Cells were then harvested and lysates were analysed by western blot.

Aggregation assay. After 24 or $48 \mathrm{~h}$ transfection aggregates were scored using a Zeiss Axiophot fluorescence microscope (Zeiss, Jena, Germany) with a $\times 25$ objective. At least 200 transfected cells in triplicate were counted in each case.

Translation. HeLa cells were transfected with vector or LRRK2 DNA. After $48 \mathrm{~h}$ of the transfection, cells were treated with $50 \mu \mathrm{g} / \mathrm{ml}$ cycloheximide (Sigma) for up to $60 \mathrm{~min}$ and then harvested for western blot analysis.

Transcription. HeLa cells were co-transfected with GFP and DsRed and either vector or LRRK2 DNA. GFP and DsRed double-positive cells were sorted and RNA was extracted using TRIZOL (Invitrogen). cDNA was generated using Superscript III Kit (Invitrogen) and qRT-PCR was performed with SYBR PCR mix (Applied Biosystems, Foster City, CA, USA) using the following primers: GFP: $5^{\prime}-A T G G$ TGAGCAAGGGCGAGGAGCT-3' (forward) 5'-GAAGATGGTGCGCTCCTGG ACGT-3' (reverse); GAPDH: $5^{\prime}$-TGTGTCCGTCGTGGATCTGA-3' (forward) $5^{\prime}$-CCTGCTTCACCACCTTCTTGAT-3' (reverse); actin: 5'-AGAAAATCTGGC ACCACACC-3' (forward) 5'-GGGGTGTTGAAGGTCTCAAA-3' (reverse)

Caspase and kinase inhibitors and antioxidant treatment. Caspase inhibitors Ac-YVAD-cho, Z-VAD-fmk and Z-DEVD-fmk (Calbiochem, Darmstadt, Germany) were used at a final concentration of $20 \mu \mathrm{M}$ in DMSO. The antioxidant $N$ Acetyl-L-cysteine (NAC) (Sigma) was dissolved in medium (DMEM), the $\mathrm{pH}$ adjusted to 7.4 and used at $1 \mathrm{mM}$ final concentration. Cells were treated after transfection and the treatment was replaced after $24 \mathrm{~h}$. For the determination of condensed nuclei and caspase 3 activation, cells were fixed $48 \mathrm{~h}$ after transfection and stained with an active caspase 3 antibody (Promega, Madison, WI, USA, 1:250). At least 200 transfected cells were scored using a Zeiss Axiophot fluorescence microscope with a $\times 63$ oil objective. Cells were treated with LRRK2 kinase inhibitors K252a (250 nM) and Raf1 kinase inhibitor I ( $2 \mu \mathrm{M})$ immediatedly after transfection for $48 \mathrm{~h}$, followed by flow cytometry.

Immunocytochemistry (ICC) and western blotting (WB). For ICC, cells were grown on coverslips coated with poly D-lysine (Sigma) and fixed for $10 \mathrm{~min}$ in $4 \%$ paraformaldehyde at room temperature. The cells were then permeabilised for $10 \mathrm{~min}$ in 1 XPBS with $2 \%$ Triton-X100, washed in 1XPBS and blocked in 1XPBS containing $1 \%$ BSA and $10 \%$ goat serum for $1 \mathrm{~h}$ at room temperature. Primary antibodies (antiFLAG 1:500 Sigma, antiHA 1:500 Sigma and antiubiquitin 1:500 Sigma) were diluted in 1XPBS with 1\%BSA and incubated overnight at $4^{\circ} \mathrm{C}$. The cells were washed in 1XPBS and incubated with the fluorescent Alexa-conjugated secondary antibody (1:500 all Invitrogen) for $1 \mathrm{~h}$ at room temperature. After two more washes, the coverslips were mounted in Fluocity containing DAPI. Images were taken with a Zeiss LSM510 confocal microscope using a $\times 63$ oil objective and analysed by Adobe Photoshop CS4 (Adobe, San Jose, CA, USA)

For WB, cells were sorted (Dako Cytomation MoFlo, Glostrup, Denmark) $48 \mathrm{~h}$ after transfection, unless otherwise indicated, harvested in RIPA buffer containing protease and phosphatase inhibitors (Roche). Protein content was determined using Bio-Rad (Hercules, CA, USA) Protein Assay kit and the proteins were separated by SDSPAGE. The blots were incubated with antibodies to antiGFP (Sigma 1:2000), antiactin (Sigma, 1:1000), antitubulin (Sigma, 1:10000), antiubiquitin (Chemicon, Rosemont, IL, USA, 1 : 5000), antiFlag (Sigma $1: 1000$ ) antiHA (Covance, Princeton, NJ, USA, 1:2000), anti $\alpha$-synuclein (Abcam, Cambridge, UK) antilC3 (Novus Biologicals, Cambridge, UK, 1:2000), antilubiquitin (Sigma, 1:5000), antiRpt5, antiRpn10, anti 4 and anti 20S (Biomol, Exeter, UK, $1: 1000$ ) in 5\% non-fat milk. The signal after the incubation with the secondary antibodies (antimouse-HRP or antirabbit-HRP Roche 1:10000) was detected by autoradiography using the Amersham (GE Healthcare, Little Chelfont, UK) chemiluminescent substrate.

Proteasome assay. Cells transfected with LRRK2, DsRed and GFP were sorted by FACS $48 \mathrm{~h}$ after transfection. DsRed and GFP double-positive cells were retained and lysed in UPS activity buffer (10 mM Tris HCl pH 7.5; 1 mM EDTA; $20 \%$ glycerol; $2 \mathrm{mM}$ ATP; 0.5\% Triton-X 100; protease inhibitor (Roche)). Proteasome activity was determined using proteasome assay buffer (Biomol) with Boc-LSTR-7AMC (Sigma), Suc-LLVY-AMC (Biomol) and Z-LLE-AMC (Biomol) as substrates for the trypsin, chymotrypsin and caspase-like activities of the proteasome, respectively. Data reflect kinetics of the linear phases of the curves of fluorigenic substrate production measured with Cytofluor multiwell plate reader (Applied Biosystems). The values from the same lysates treated with $10 \mu \mathrm{M}$ MG132 correspond to non-proteasomal proteases and were subtracted to the proteasome activity values.

Zebrafish model: maintenance of stocks and collection of embryos. Wild-type zebrafish of the $A B$ strain was reared under standard conditions. ${ }^{39}$ Embryos were collected from natural spawnings, staged according to established criteria ${ }^{40}$ and reared in embryo medium $(5 \mathrm{mM} \mathrm{NaCl}, 0.17 \mathrm{mM} \mathrm{KCl}$, $0.33 \mathrm{mM} \mathrm{CaCl}_{2}, 0.33 \mathrm{mM} \mathrm{Mg}_{2} \mathrm{SO}_{4}, 10-5 \%$ Methylene Blue).

Zebrafish model: transient DNA overexpression. Injection solutions were prepared using $100 \mu \mathrm{g} / \mathrm{ml}$ of linearised plasmid DNA (either WTLRRK2, GSLRRK2, LRRK1 or vector) with $50 \mu \mathrm{g} / \mathrm{ml}$ of linearised plasmid Ub ${ }^{\mathrm{G} 76 \mathrm{~V}}$-GFP. Approximately $2 \mathrm{nl}$ of the injection solution was injected into the yolk of embryos at the 1-2 cell stage. Embryos were viewed at regular intervals using an Olympus SZX12 dissecting stereomicroscope (Olympus, Tokyo, Japan) under epifluorescence with GFP filters. The number of embryos with mosaic GFP expression was counted at regular intervals between 12 and 30 h.p.f. Digital images were captured using an Olympus DP12 digital camera. For western blotting, embryos were manually dechorionated and homogenised in lysis buffer then processed as described above.

Statistics. In the case of flow cytometry, experiments were performed three times independently in triplicates. Due to slightly different flow cytometer settings on different days and different transfection efficiencies, fluorescence levels of control cells vary largely between independent experiments. The statistical analysis of independent data from different days is therefore difficult. Instead, we have analysed the replicate data in each separate experiment by two-tailed, unpaired Student's $t$-test and shown $P$-values that are representative for all independent experiments. In order to graphically display data of three independent experiments in one combined graph, data of each separate experiment were normalised to the mean of the respective control samples. The mean of all replicates was calculated and is shown \pm S.D. of all replicates. In the case of immunocytochemnistry, experiments were also performed three times in triplicates, and data are shown as the means of three independent experiments \pm S.E.M. $P$-values were calculated by unconditional logistical regression analysis using the general log linear analysis option of SPSS version 6.1 software (IBM, Armonk, NY, USA). For the statistical analysis of western blot data, two-tailed paired Student's t-test was performed on raw densitometry data from at least three independent experiments. 


\section{Conflict of Interest}

The authors declare no conflict of interest.

Acknowledgements. We thank Marie Quick, Katie Woodhouse, Adrian McNabb and Tomasz Dyl for technical support. This work was further supported by the Flow Cytometry Core facility at the CIMR (Wellcome Trust Strategic Award No. 079895), and in particular we wish to thank A Petrunkina-Harrison for her advice and support. We thank N Dantuma, CA Ross, MR Cookson, DR Alessi and C Patterson for constructs and N Mizushima for the Atg5 knockout cell line. This work was supported by The Wellcome Trust (Senior Clinical Fellowship to DCR), the Medical Research Council, the FECYT, a Wellcome Trust/Medical Research Council Strategic Award in Neurodegeneration, NIHR Biomedical Research Centre at Addenbrooke's Hospital, and the European Community's Seventh Framework Programme (FP7/2007-2013) under Grant agreement number 241791 (MEFOPA). The funders had no role in study design, data collection and analysis, decision to publish or preparation of the manuscript.

1. Dauer W, Przedborski S. Parkinson's disease: mechanisms and models. Neuron 2003; 39 : 889-909.

2. Singleton $A B$, Farrer $M$, Johnson J, Singleton A, Hague $S$, Kachergus $J$ et al. alphaSynuclein locus triplication causes Parkinson's disease. Science 2003; 302: 841.

3. Zimprich A, Biskup S, Leitner P, Lichtner P, Farrer M, Lincoln S et al. Mutations in LRRK2 cause autosomal-dominant parkinsonism with pleomorphic pathology. Neuron 2004; 44 : 601-607.

4. Paisan-Ruiz C, Jain S, Evans EW, Gilks WP, Simon J, van der Brug M et al. Cloning of the gene containing mutations that cause PARK8-linked Parkinson's disease. Neuron 2004; 44: $595-600$.

5. Adams JR, van Netten H, Schulzer M, Mak E, McKenzie J, Strongosky A et al. PET in LRRK2 mutations: comparison to sporadic Parkinson's disease and evidence for presymptomatic compensation. Brain 2005; 128 (Part 12): 2777-2785.

6. Mata IF, Wedemeyer WJ, Farrer MJ, Taylor JP, Gallo KA. LRRK2 in Parkinson's disease: protein domains and functional insights. Trends Neurosci 2006; 29: 286-293.

7. Lin X, Parisiadou L, Gu XL, Wang L, Shim H, Sun L et al. Leucine-rich repeat kinase 2 regulates the progression of neuropathology induced by Parkinson's-disease-related mutant alpha-synuclein. Neuron 2009; 64: 807-827.

8. Andres-Mateos E, Mejias R, Sasaki M, Li X, Lin BM, Biskup S et al. Unexpected lack of hypersensitivity in LRRK2 knock-out mice to MPTP (1-methyl-4-phenyl-1,2,3,6tetrahydropyridine). J Neurosci 2009; 29: 15846-15850.

9. West AB, Moore DJ, Biskup S, Bugayenko A, Smith WW, Ross CA et al. Parkinson's disease-associated mutations in leucine-rich repeat kinase 2 augment kinase activity. Proc Natl Acad Sci USA 2005; 102: 16842-16847.

10. West AB, Moore DJ, Choi C, Andrabi SA, Li X, Dikeman D et al. Parkinson's diseaseassociated mutations in LRRK2 link enhanced GTP-binding and kinase activities to neuronal toxicity. Hum Mol Genet 2007; 16: 223-232.

11. Cookson MR, Dauer W, Dawson T, Fon EA, Guo M, Shen J. The roles of kinases in familial Parkinson's disease. J Neurosci 2007; 27: 11865-11868.

12. Smith WW, Pei Z, Jiang H, Dawson VL, Dawson TM, Ross CA. Kinase activity of mutant LRRK2 mediates neuronal toxicity. Nat Neurosci 2006; 9: 1231-1233.

13. Greggio E, Jain S, Kingsbury A, Bandopadhyay R, Lewis $P$, Kaganovich $A$ et al. Kinase activity is required for the toxic effects of mutant LRRK2/dardarin. Neurobiol Dis 2006; 23: 329-341.

14. MacLeod D, Dowman J, Hammond R, Leete T, Inoue K, Abeliovich A. The familial Parkinsonism gene LRRK2 regulates neurite process morphology. Neuron 2006; 52 : 587-593.

15. Korr $D$, Toschi L, Donner $P$, Pohlenz HD, Kreft B, Weiss $B$. LRRK1 protein kinase activity is stimulated upon binding of GTP to its Roc domain. Cell Signal 2006; 18: 910-920.

16. Narain $Y$, Wyttenbach A, Rankin J, Furlong RA, Rubinsztein DC. A molecular investigation of true dominance in Huntington's disease. J Med Genet 1999; 36: 739-746.

17. Korolchuk VI, Mansilla A, Menzies FM, Rubinsztein DC. Autophagy inhibition compromises degradation of ubiquitin-proteasome pathway substrates. Mol Cell 2009; 33: 517-527.

18. Carballo-Carbajal I, Weber-Endress S, Rovelli G, Chan D, Wolozin B, Klein CL et al. Leucine-rich repeat kinase 2 induces alpha-synuclein expression via the extracellular signal-regulated kinase pathway. Cell Signal 2010; 22: 821-827.
19. Mizushima N, Yamamoto A, Hatano M, Kobayashi Y, Kabeya Y, Suzuki K et al. Dissection of autophagosome formation using Apg5-deficient mouse embryonic stem cells. J Cell Biol 2001; 152: 657-668.

20. Dantuma NP, Lindsten K, Glas R, Jellne M, Masucci MG. Short-lived green fluorescent proteins for quantifying ubiquitin/proteasome-dependent proteolysis in living cells. Nat Biotechnol 2000; 18: 538-543.

21. laccarino C, Crosio C, Vitale C, Sanna G, Carri MT, Barone P. Apoptotic mechanisms in mutant LRRK2-mediated cell death. Hum Mol Genet 2007; 16: 1319-1326.

22. Gloeckner CJ, Kinkl N, Schumacher A, Braun RJ, O'Neill E, Meitinger T et al. The Parkinson disease causing LRRK2 mutation I2020T is associated with increased kinase activity. Hum Mol Genet 2006; 15: 223-232.

23. Anand VS, Reichling LJ, Lipinski K, Stochaj W, Duan W, Kelleher K et al. Investigation of leucine-rich repeat kinase 2: enzymological properties and novel assays. Febs J 2009; 276: 466-478.

24. Covy JP, Giasson BI. Identification of compounds that inhibit the kinase activity of leucinerich repeat kinase 2. Biochem Biophys Res Commun 2009; 378: 473-477.

25. Beere HM, Wolf BB, Cain K, Mosser DD, Mahboubi A, Kuwana T et al. Heat-shock protein 70 inhibits apoptosis by preventing recruitment of procaspase- 9 to the Apaf-1 apoptosome. Nat Cell Biol 2000; 2: 469-475.

26. Jaleel M, Nichols RJ, Deak M, Campbell DG, Gillardon F, Knebel A et al. LRRK2 phosphorylates moesin at threonine-558: characterization of how Parkinson's disease mutants affect kinase activity. Biochem J 2007; 405: 307-317.

27. Lin X, Parisiadou L, Gu X-L, Wang L, Shim H, Sun L et al. Leucine-rich repeat kinase 2 regulates the progression of neuropathology induced by Parkinson's disease-related mutant alpha-synuclein. Neuron 2009; 64: 807-827.

28. Ishihara L, Warren L, Gibson R, Amouri R, Lesage S, Durr A et al. Clinical features of Parkinson disease patients with homozygous leucine-rich repeat kinase 2 G2019S mutations. Arch Neurol 2006; 63: 1250-1254.

29. Liu Z, Wang X, Yu Y, Li X, Wang T, Jiang $\mathrm{H}$ et al. A Drosophila model for LRRK2-linked parkinsonism. Proc Natl Acad Sci USA 2008; 105: 2693-2698.

30. $\mathrm{Ng} \mathrm{CH}$, Mok SZ, Koh C, Ouyang X, Fivaz ML, Tan EK et al. Parkin protects against LRRK2 G2019S mutant-induced dopaminergic neurodegeneration in Drosophila. J Neurosci 2009; 29: 11257-11262.

31. Venderova K, Kabbach G, Abdel-Messih E, Zhang Y, Parks RJ, Imai Y et al. Leucine-rich repeat kinase 2 interacts with Parkin, DJ-1 and PINK- 1 in a Drosophila melanogaster model of Parkinson's disease. Hum Mol Genet 2009; 18: 4390-4404.

32. Li X, Patel JC, Wang J, Avshalumov MV, Nicholson C, Buxbaum JD et al. Enhanced striatal dopamine transmission and motor performance with LRRK2 overexpression in mice is eliminated by familial Parkinson's disease mutation G2019S. J Neurosci 2010; 30: 1788-1797.

33. Li Y, Liu W, Oo TF, Wang L, Tang Y, Jackson-Lewis V et al. Mutant LRRK2(R1441G) BAC transgenic mice recapitulate cardinal features of Parkinson's disease. Nat Neurosci 2009; 12: $826-828$

34. Biskup S, Moore DJ, Celsi F, Higashi S, West AB, Andrabi SA et al. Localization of LRRK2 to membranous and vesicular structures in mammalian brain. Ann Neurol 2006; 60: 557-569.

35. Hatano T, Kubo S, Imai S, Maeda M, Ishikawa K, Mizuno $Y$ et al. Leucine-rich repeat kinase 2 associates with lipid rafts. Hum Mol Genet 2007; 16: 678-690.

36. Greggio E, Lewis PA, van der Brug MP, Ahmad R, Kaganovich A, Ding J et al. Mutations in LRRK2/dardarin associated with Parkinson disease are more toxic than equivalent mutations in the homologous kinase LRRK1. J Neurochem 2007; 102: 93-102.

37. Smith WW, Pei Z, Jiang H, Moore DJ, Liang Y, West AB et al. Leucine-rich repeat kinase 2 (LRRK2) interacts with parkin, and mutant LRRK2 induces neuronal degeneration. Proc Natl Acad Sci USA 2005; 102: 18676-18681.

38. Furlong RA, Narain Y, Rankin J, Wyttenbach A, Rubinsztein DC. alpha-synuclein overexpression promotes aggregation of mutant huntingtin. Biochem J 2000; 346 (Part 3): $577-581$.

39. Westerfield M. A guide for the Laboratory Use of Zebrafish (Danio rerio). University of Oregon Press: Eugene, Oregon, 1995.

40. Kimmel CB, Ballard WW, Kimmel SR, Ullmann B, Schilling TF. Stages of embryonic development of the zebrafish. Dev Dyn 1995; 203: 253-310.

Cell Death and Disease is an open-access journal published by Nature Publishing Group. This work is licensed under the Creative Commons Attribution-Noncommercial-No Derivative Works 3.0 Unported License. To view a copy of this license, visit http://creativecommons.org/licenses/by-nc-nd/3.0/

\section{Supplementary Information accompanies the paper on Cell Death and Disease website (http://www.nature.com/cddis)}

